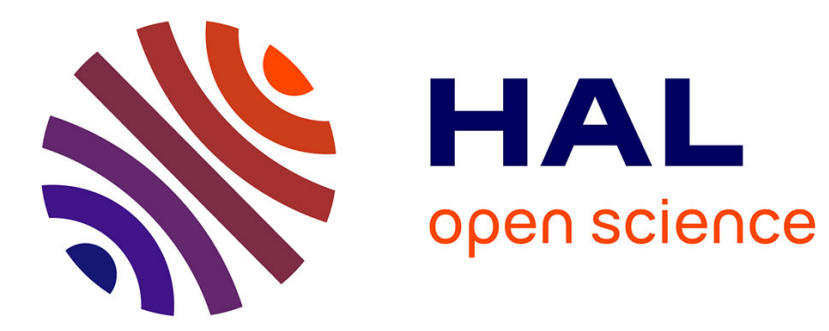

\title{
Carrier dynamics near a crack in GaN microwires with AlGaN multiple quantum wells
}

Sylvain Finot, Vincent Grenier, Vitaly Zubialevich, Catherine Bougerol, Pietro Pampili, Joël Eymery, Peter Parbrook, Christophe Durand, Gwenolé Jacopin

\section{- To cite this version:}

Sylvain Finot, Vincent Grenier, Vitaly Zubialevich, Catherine Bougerol, Pietro Pampili, et al.. Carrier dynamics near a crack in GaN microwires with AlGaN multiple quantum wells. Applied Physics Letters, 2020, 117 (22), pp.221105. 10.1063/5.0023545 . hal-03081456

\section{HAL Id: hal-03081456 https://hal.science/hal-03081456}

Submitted on 18 Dec 2020

HAL is a multi-disciplinary open access archive for the deposit and dissemination of scientific research documents, whether they are published or not. The documents may come from teaching and research institutions in France or abroad, or from public or private research centers.
L'archive ouverte pluridisciplinaire HAL, est destinée au dépôt et à la diffusion de documents scientifiques de niveau recherche, publiés ou non, émanant des établissements d'enseignement et de recherche français ou étrangers, des laboratoires publics ou privés. 


\title{
Carrier Dynamics near a Crack in GaN microwires with AIGaN multiple quantum wells
}

\author{
Sylvain Finot, ${ }^{1, \text { a) }}$ Vincent Grenier, ${ }^{2}$ Vitaly Zubialevich, ${ }^{3}$ Catherine Bougerol, ${ }^{1}$ Pietro Pampili, ${ }^{3}$ Joël Eymery, ${ }^{4}$ \\ Peter J. Parbrook, ${ }^{3}$ Christophe Durand, ${ }^{2}$ and Gwénolé Jacopin ${ }^{1}$ \\ 1) Univ. Grenoble Alpes, CNRS, Grenoble INP*, Institut Néel, 38000 Grenoble, \\ France \\ 2) Univ. Grenoble Alpes, CEA, IRIG, PHELIQS, NPSC, 38000 Grenoble, France \\ 3) Tyndall National Institute, University College Cork, Cork T12 R5CP, Ireland \\ ${ }^{4)}$ Univ. Grenoble Alpes, CEA, IRIG, MEM, NRS, 38000 Grenoble, France \\ (Dated: 28 October 2020)
}

Relaxation of tensile strain in AlGaN heterostructures grown on GaN template can lead to the formation of cracks. These extended defects locally degrade the crystal quality resulting in a local increase of non-radiative recombinations. The effect of such cracks on the optical and structural properties of core-shell $\mathrm{AlGaN} / \mathrm{AlGaN}$ multiple quantum wells grown on GaN microwires are comprehensively characterized by means of spectrally and time-correlated cathodoluminescence (CL). We observe that the CL blueshifts near a crack. By performing $6 \mathrm{x} 6 \mathrm{k}$.p simulations in combination with transmission electron microscopy analysis, we ascribe this shift to the strain relaxation by the free surface near cracks. By simultaneously recording the variations of both the CL lifetime and the CL intensity across the crack, we directly assess the carrier dynamics around the defect at $T=5 \mathrm{~K}$. We observe that the CL lifetime is reduced typically from 500 ps to less than $300 \mathrm{ps}$ and the CL intensity increases by about $40 \%$ near the crack. The effect of the crack on the optical properties is therefore of two natures. First, the presence of this defect locally increases non-radiative recombinations while at the same time, it locally improves the extraction efficiency. These findings emphasize the need for time-resolved experiments to avoid experimental artifacts related to local changes of light collection.

Efficient ultra-violet (UV) light sources are used in many applications related to spectroscopy, photopolymer curing, water purification, medical treatment and disinfection. Compared to traditional UV lamps, III-nitride UV light emitting diodes (LEDs) present a number of advantages such as compactness, long lifetime and are $\mathrm{Hg}$ free. ${ }^{1}$ However, despite a significant research effort in the past two decades, the external quantum efficiency (EQE) remains rather low (below $20 \%$ in the UV-C range $^{2}$ ). The factors limiting their EQE are manifold: influence of extended and point defects, ${ }^{3}$ poor p-doping efficiency ${ }^{4}$ and modest light extraction. ${ }^{5}$

To overcome some of the inherent limitations of planar $c$-plane LED architectures, the use of nanowire-based UV LEDs has recently emerged. ${ }^{6}$ They already offer some key advantages: higher $\mathrm{Mg}$ solubility leading to a more efficient p-doping in the deep- $\mathrm{UV}^{7,8}$ and a drastic reduction of extended defects. ${ }^{9}$ A particularly promising approach is based on $m$-plane core-shell wires. ${ }^{10-15}$ In this geometry, the absence of the quantum confined Stark effect and the increased emitting area contribute to the mitigation of the detrimental effects that appear at high carrier densities. Furthermore, core-shell wires have already been used to develop flexible LEDs, ${ }^{16}$ this configuration could then lead to flexible UV LEDs.

In a core-shell microwire, the emitting dipole is usually coupled to a guided mode where the wire itself acts as a waveguide. ${ }^{17}$ As a consequence, for UV emitters, the light extraction could be severely altered due to the

a)sylvain.finot@neel.cnrs.fr detrimental GaN core parasitic absorption. To circumvent this issue, one approach is to use an AlN inner core. ${ }^{10}$ However, with such a design, the electrical injection is challenging. Hence, to combine electrical injection and low absorption losses, a solution is to grow an AlGaN cladding shell to protect the guided mode from inner core absorption losses. To be efficient in the deep UV emission, this cladding layer has to be both Al-rich and thick enough. Under these conditions, and as already observed for planar layers, this often leads to crack formation. ${ }^{18}$ Therefore, it is crucial to investigate the influence of cracks on the optical properties of the active region. To do so, a preferred technique is cathodoluminescence (CL) spectroscopy. ${ }^{19,20}$ However, CL spectroscopy does not allow to separate changes in light extraction efficiency (LEE) and in internal quantum efficiency (IQE). To overcome these limitations, time-resolved CL (TRCL) techniques have been proposed. ${ }^{21,22}$

Here, using a spatially resolved time-correlated CL (SRTC-CL) setup, ${ }^{23,24}$ we probe the carrier dynamics near cracks in core-shell GaN/AlGaN microwires with a spatial resolution of $50 \mathrm{~nm}$ and a temporal resolution better than 40 ps (see supp. material Sec. III for details about the timing resolution estimation). We obtain the distributions of emission peak energies, CL lifetimes, and CL intensities around such a defect. By comparing our experimental data with a 6x6 k.p Schrödinger simulations, we are able to explain the observed behaviors. These results give precious information about the carrier dynamics around cracks and confirm the relevance of such a technique to elucidate the influence of defects on III-nitride nanostructures. 


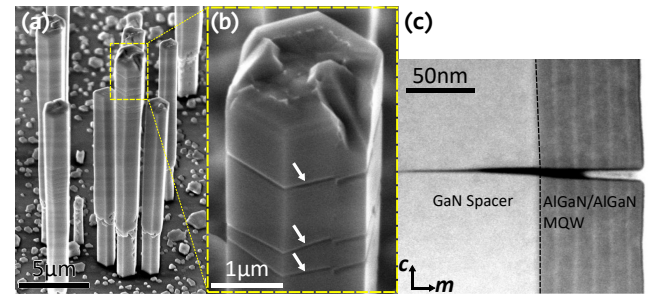

FIG. 1. (a)-(b) $45^{\circ}$-tilted SEM image of GaN wires covered with Al-based MQWs grown on $c$-sapphire substrate. (b) Enlarged view near the wire top with presence of multiple cracks. (c) HAADF-STEM longitudinal cross-section near a radial crack.

The studied GaN microwires with core-shell AlGaN heterostructure were grown on $c$-plane sapphire substrates by metalorganic vapor-phase epitaxy (MOVPE). The wires are mainly N-polar but may exhibit polarity mixing. The growth is initiated with a heavily ndoped $\left(10^{20} \mathrm{~cm}^{-3}\right) \mathrm{GaN}$ wire core grown under high silane flux $\left(\approx 200 \mathrm{nmol} \cdot \mathrm{min}^{-1}\right)$, which favors the vertical growth. ${ }^{25}$ Then, an undoped GaN section is grown. The shell, which preferentially grows on the undoped GaN core, starts from an unintentionally doped GaN spacer $(\approx 150 \mathrm{~nm})$ to bury the contamination surface layer. ${ }^{26}$ Then, the active region containing five $\mathrm{AlGaN} / \mathrm{AlGaN}$ quantum wells (QWs) is grown at $950^{\circ} \mathrm{C}$ under $\mathrm{N}_{2}$ (100 mbar) with a $\mathrm{V} / \mathrm{III}$ ratio close to 1000 . The $\mathrm{Al}$ content in the barrier has been estimated to be around 70 $\pm 10 \%$, while the $\mathrm{Al}$ content is close to $45 \pm 5 \%$ in QWs (estimation based on X-ray diffraction performed on $c$ plane AlGaN layers grown under identical conditions). ${ }^{27}$ Figure 1 (a) displays a $45^{\circ}$-tilted scanning electron microscopy (SEM) image of typical as-grown wires. Due to the large lattice mismatch between the GaN core and the Al-rich AlGaN shell, we observe the formation of cracks for most of the wires (Figure 1(b)).

In a core-shell geometry, the strain is mainly enhanced along the $c$-axis $\left(\epsilon_{z z}\right) \cdot{ }^{14,28}$ For this reason, these cracks preferentially form perpendicular to the $c$-axis, along the $a$-axis. To precisely determine the structural properties of these defects, longitudinal cross-sections have been prepared by focused Ga-ion beam using a STRATA 400S equipment and observed by scanning transmission electron microscopy (STEM) using a FEI-Tecnai microscope operated at $200 \mathrm{kV}$. Figure $1(\mathrm{c})$ shows a cross-section STEM image allowing to deduce the QW and barrier thicknesses, respectively equal to $4 \pm 1 \mathrm{~nm}$ and $10 \pm 1 \mathrm{~nm}$ We observe that the crack runs through the entire AlGaN heterostructure and terminates in the GaN spacer. The thickness of the heterostructure does not seem to be affected by the presence of the crack. EDX measurements have also been performed, showing no significant variation in the Al-content (see supp. material Sec. VII). This suggests that cracks appear at the end of the growth or upon cooldown. ${ }^{29}$

To probe the influence of cracks on the optical properties, we performed 5K-CL measurements. First, microwires are mechanically dispersed on $\mathrm{SiO}_{2} / \mathrm{Si}$ substrates. For each wire, CL spectra are acquired in the $3.7-4.9 \mathrm{eV}$ energy range with a $2 \mathrm{kV}$ acceleration voltage and a dwell time of $10 \mathrm{~ms}$. Monte Carlo simulations allow us to estimate the statistical distribution of the electron penetration depth. ${ }^{30}$ In this given configuration, only the first three QWs are expected to contribute to the luminescence (detailed in supp. material Sec. V). An SEM image of a typical wire with multiple cracks is shown in Figure 2(a). Figure 2(b) shows the corresponding CL map integrated over the wire's width in the MQWs energy region. The MQWs emission energy is slightly shifting to higher emission energy from bottom to the top probably due to an $\mathrm{Al}$ gradient along the wire or a change in QWs thickness. Even if cracks can be difficult to locate on SEM images, they can easily be seen on the CL spectra map. In fact, the presence of a crack systematically leads to an energy blue-shift of about $100 \mathrm{meV}$ associated with a significant increase of CL intensity (see supp. materials sec IV for high resolution monochromatic CL images).

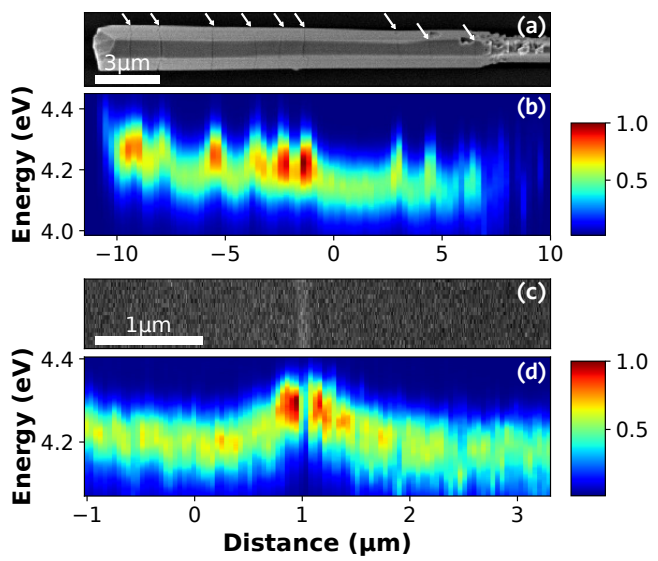

FIG. 2. (a) SEM image of a full wire and (b) the corresponding mapping of the CL spectra along the wire.(c) Close-view SEM image near a crack on a different wire and its corresponding CL spectra map (d). The measurements were done at $5 \mathrm{~K}$ with a $5 \mathrm{kV}$ acceleration voltage for (a) and (b) and $2 \mathrm{kV}$ for (c) and (d). The horizontal axis corresponds to the $c$-axis. The colorbars indicate the normalized CL intensity.

We compared our observations with two-dimensional 6x6 k.p simulations performed with the nextnano3 software. ${ }^{31}$ The parameters used can be found in ref 32 . The simulated system is a replica of the observed stacking in the TEM cross-sections (Figure 1(c)). To reproduce the experimental transition energies, the $\mathrm{Al}$ content in the MQWs has been adjusted to $40 \%$ and $60 \%$ in the 


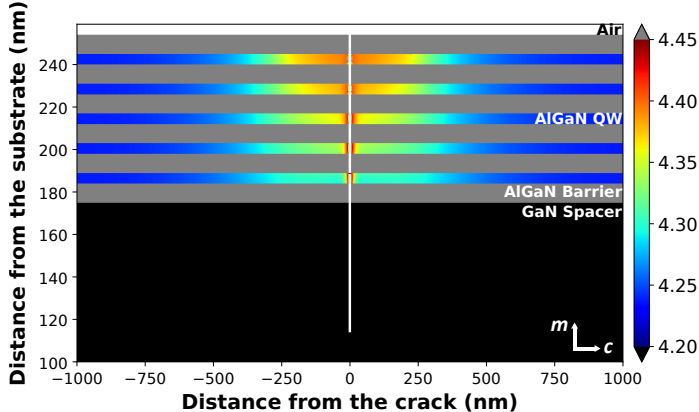

(a)
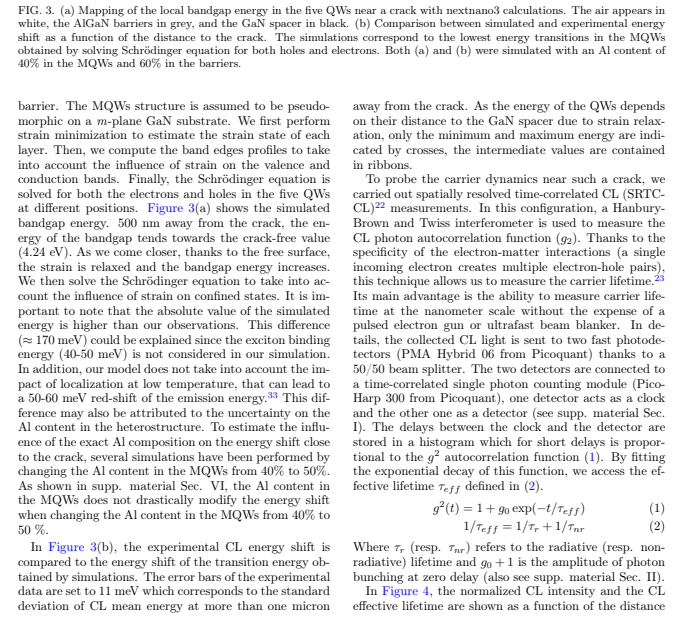

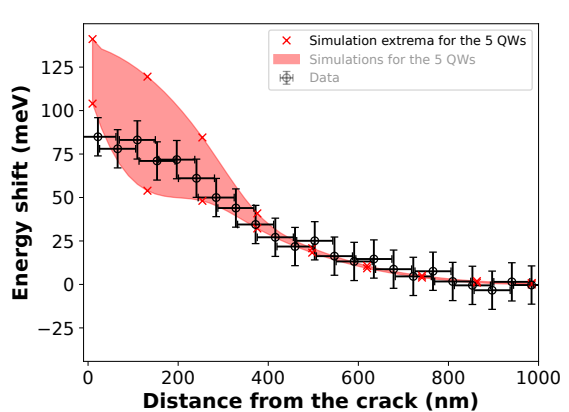

(b) 
to the crack. About $400 \mathrm{~nm}$ away from the crack, the CL intensity starts to increase to reach 1.4 times the intensity of the crack-free region at $100 \mathrm{~nm}$. Under $100 \mathrm{~nm}$, the intensity quickly decreases to its minimum, which corresponds to 0.7 times the crack-free region. The lifetime is strictly decreasing with the distance, it ranges from $475 \mathrm{ps}$ at one micron away from crack to $285 \mathrm{ps}$ near the crack.

We can consider that this lifetime reduction is driven either by the radiative or the non-radiative lifetime. The first hypothesis is very unlikely. Indeed, assuming that only the radiative lifetime is modified, it is possible to extract the non-radiative lifetime $\tau_{n r}=920 \pm 88 \mathrm{ps}$, the radiative lifetime near the crack $\tau_{r, \text { crack }}=410 \pm 42 \mathrm{ps}$ and far from the crack $\tau_{r, b u l k}=980 \pm 135$ ps. Such an improvement would correspond to a significant increase in oscillator strength (more than a factor of 2), which is not seen in our simulations.

Therefore, the reduction of effective lifetime near the crack is assigned to the increasing influence of nonradiative recombinations near the surface. This lifetime reduction could originate from the strong electric field at the surface due to Fermi level pinning and piezoelectric fields. ${ }^{34}$ Such a field dissociates excitons and spatially separates electron-hole pairs, preventing them from radiative recombinations. This lifetime shortening could also be linked to the formation of non-radiative centers in the near-surface region associated with crack generation. In addition, the increase in CL intensity is most likely due to a local improvement of LEE. This local LEE

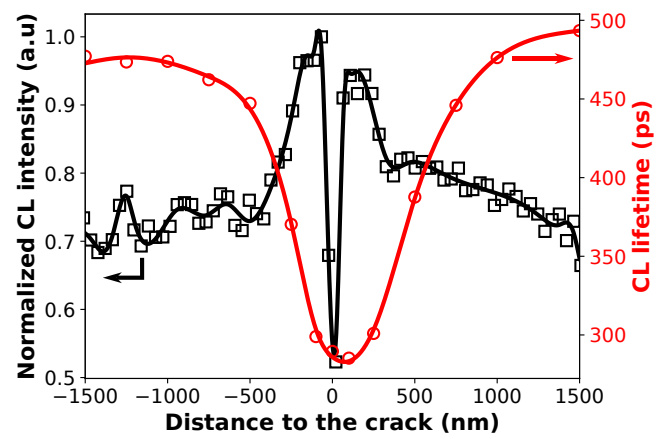

FIG. 4. Normalized CL intensity of the MQW (black squares) and CL lifetime (red circles) as a distance to the crack acquired at $\mathrm{T}=5 \mathrm{~K}$. The lifetime has been acquired with a SRTC-CL setup.

increase has two origins. First, by locally breaking the symmetry of the structure, the crack could act as a scattering center for the emitted light, which thus offers a possible coupling to the free space. Due to the GaN core parasitic absorption $\left(\alpha_{\mathrm{GaN}}(4.2-4.4 \mathrm{eV}) \approx 2.10^{5} \mathrm{~cm}^{-1}\right){ }^{35}$ UV photons emitted in the wire can only propagate on few hundreds of nanometers. As a consequence, only the light generated close to the crack would benefit from a better extraction efficiency, which is consistent with our observations (see Figure 4). Second, as evidenced by our simulations (not shown here), the change of strain state near the crack induces a reordering of the valence bands. ${ }^{36,37}$ Due to their different selection rules, it should result in a change of polarization direction of the emitted light. Since LEE drastically depends on the light polarization, ${ }^{1}$ such crossover could locally increase the observed CL intensity. To further confirm this hypothesis, polarization-dependent CL measurements should be performed. ${ }^{38}$

In conclusion, by probing the optical properties of GaN wires with Al-rich core-shell MQWs using SRTC-CL, we demonstrate the influence of cracks on the efficiency of the active region. Driven by strain relaxation, the MQWs emission energy increases near the crack $(\approx 100 \mathrm{meV})$. In addition, we observe an increase of CL intensity near the crack that is assigned to a local increase of the LEE. On the other hand, the carrier lifetime experiences a pronounced decrease close to the extended defect due to non-radiative recombinations that happen near the crack surface. Our results emphasize the need for time-resolved CL to reveal the change, at the nanometer scale, in IQE without being affected by local changes of LEE.

See the supplementary materials for a description of (I) the experimental setup, (II) the SRTC-CL operation principle, (III) time resolution estimation, (IV) additional CL measurements, (V) Casino simulations, (VI) the effect of $\mathrm{Al}$ content on the transition emission energy and (VII) EDX measurements.

This work has been financially supported by the program "Initiatives de Recherche Stratégiques" (IRS) of IDEX Univ. Grenoble Alpes (15-IDEX-0002), by the French-Irish program ULYSSES administered by the Irish Research Council and Campus-France and by Science Foundation Ireland through awards 18/TIDA/6066 and $12 / \mathrm{RC} / 2276 \_\mathrm{P} 2$. The authors would like to thank Fabrice Donatini for his help in the development of the SRTC-CL setup and Hanako Okuno for her help with the EDX measurements.

\section{DATA AVAILABILITY}

The data that support the findings of this study are available from the corresponding authors upon reasonable request.

${ }^{1}$ M. Kneissl, III-Nitride Ultraviolet Emitters, edited by M. Kneissl and J. Rass, Springer Series in Materials Science, Vol. 227 (Springer International Publishing, Cham, 2016).

${ }^{2}$ T. Takano, T. Mino, J. Sakai, N. Noguchi, K. Tsubaki, and H. Hirayama, "Deep-ultraviolet light-emitting diodes with external quantum efficiency higher than $20 \%$ at $275 \mathrm{~nm}$ achieved by improving light-extraction efficiency," Applied Physics Express 10, 31002 (2017)

${ }^{3}$ M. Shatalov, W. Sun, A. Lunev, X. Hu, A. Dobrinsky, Y. Bilenko, J. Yang, M. Shur, R. Gaska, C. Moe, G. Garrett, and M. Wraback, "AlGaN Deep-Ultraviolet Light-Emitting Diodes 
with External Quantum Efficiency above 10\%," Applied Physics Express 5, 82101 (2012).

${ }^{4}$ Y. Taniyasu, M. Kasu, and T. Makimoto, "An aluminium nitride light-emitting diode with a wavelength of 210 nanometres," Nature 441, 325-328 (2006).

${ }^{5}$ M. Kneissl, T.-Y. Seong, J. Han, and H. Amano, "The emergence and prospects of deep-ultraviolet light-emitting diode technologies," Nature Photonics 13, 233-244 (2019).

${ }^{6}$ S. Zhao, A. T. Connie, M. H. T. Dastjerdi, X. H. Kong, Q. Wang M. Djavid, S. Sadaf, X. D. Liu, I. Shih, H. Guo, and Z. Mi, "Aluminum nitride nanowire light emitting diodes: Breaking the fundamental bottleneck of deep ultraviolet light sources," Scientific Reports 5, 8332 (2015)

${ }^{7}$ A. M. Siladie, G. Jacopin, A. Cros, N. Garro, E. Robin, D. Caliste, P. Pochet, F. Donatini, J. Pernot, and B. Daudin, "Mg and in Codoped p-type AlN Nanowires for pn Junction Realization," Nano Letters 19, 8357-8364 (2019).

${ }^{8}$ N. H. Tran, B. H. Le, S. Zhao, and Z. Mi, "On the mechanism of highly efficient p-type conduction of Mg-doped ultra-widebandgap AlN nanostructures," Applied Physics Letters 110 32102 (2017).

${ }^{9}$ O. Landré, D. Camacho, C. Bougerol, Y. M. Niquet, V. FavreNicolin, G. Renaud, H. Renevier, and B. Daudin, "Elastic strain relaxation in GaN/AlN nanowire superlattice," Physical Review B - Condensed Matter and Materials Physics 81, 1-4 (2010).

${ }^{10}$ P.-M. Coulon, G. Kusch, R. W. Martin, and P. A. Shields, "Deep UV Emission from Highly Ordered AlGaN/AlN Core-Shell Nanorods," ACS Applied Materials \& Interfaces 10, 33441-33449 (2018).

${ }^{11}$ Q. Wang, H. P. T. Nguyen, K. Cui, and Z. Mi, "High efficiency ultraviolet emission from $\mathrm{Al} \times \mathrm{Ga}$ 1-x $\mathrm{N}$ core-shell nanowire heterostructures grown on $\mathrm{Si}$ (111) by molecular beam epitaxy," Applied Physics Letters 101, 043115 (2012).

${ }_{12}$ M. D. Brubaker, K. L. Genter, A. Roshko, P. T. Blanchard, B. T. Spann, T. E. Harvey, and K. A. Bertness, "UV LEDs based on $\mathrm{p}-\mathrm{i}-\mathrm{n}$ core-shell $\mathrm{AlGaN} / \mathrm{GaN}$ nanowire heterostructures grown by N-polar selective area epitaxy," Nanotechnology 30, 234001 (2019).

${ }^{13}$ P.-M. Coulon, G. Kusch, E. D. Le Boulbar, P. Chausse, C. Bryce, R. W. Martin, and P. A. Shields, "Hybrid Top-Down/BottomUp Fabrication of Regular Arrays of AlN Nanorods for Deep-UV Core-Shell LEDs," physica status solidi (b) 255, 1700445 (2018).

${ }^{14}$ C. Durand, C. Bougerol, J.-F. Carlin, G. Rossbach, F. Godel, J. Eymery, P.-H. Jouneau, A. Mukhtarova, R. Butté, and N. Grandjean, "M -Plane GaN/InAlN Multiple Quantum Well in Core-Shell Wire Structure for UV Emission," ACS Photonics 1, 38-46 (2014).

${ }^{15}$ V. Grenier, S. Finot, G. Jacopin, C. Bougerol, E. Robin, N. Mollard, B. Gayral, E. Monroy, J. Eymery, and C. Durand, "UV Emission from GaN Wires with $\mathrm{m}$-Plane Core-Shel GaN/AlGaN Multiple Quantum Wells ," ACS Applied Materials \& Interfaces 12, 44007-44016 (2020)

${ }^{16} \mathrm{X}$. Dai, A. Messanvi, H. Zhang, C. Durand, J. Eymery, C. Bougerol, F. H. Julien, and M. Tchernycheva, "Flexible LightEmitting Diodes Based on Vertical Nitride Nanowires," Nano Letters 15, 6958-6964 (2015).

${ }^{17} \mathrm{M}$. Tchernycheva, A. Messanvi, A. de Luna Bugallo, G. Jacopin, P. Lavenus, L. Rigutti, H. Zhang, Y. Halioua, F. H. Julien, J. Eymery, and C. Durand, "Integrated Photonic Platform Based on InGaN/GaN Nanowire Emitters and Detectors," Nano Letter 14, 3515-3520 (2014).

${ }^{18}$ S. Einfeldt, V. Kirchner, H. Heinke, M. Dießelberg, S. Figge, K. Vogeler, and D. Hommel, "Strain relaxation in AlGaN under tensile plane stress," Journal of Applied Physics 88, 7029-7036 (2000).

${ }^{9}$ S. Einfeldt, M. Dießelberg, H. Heinke, D. Hommel, D. Rudloff, J. Christen, and R. F. Davis, "Strain in cracked AlGaN layers," Journal of Applied Physics 92, 118-123 (2002).

${ }^{20}$ D. Rudloff, T. Riemann, J. Christen, Q. K. K. Liu, A. Kaschner, A. Hoffmann, C. Thomsen, K. Vogeler, M. Diesselberg, S. Ein- feldt, and D. Hommel, "Stress analysis of AlxGa1-xN films with microcracks," Applied Physics Letters 82, 367-369 (2003)

${ }^{21}$ W. Liu, J.-F. Carlin, N. Grandjean, B. Deveaud, and G. Jacopin, "Exciton dynamics at a single dislocation in GaN probed by picosecond time-resolved cathodoluminescence," Applied Physics Letters 109, 042101 (2016).

${ }^{22}$ S. Meuret, L. H. G. Tizei, T. Auzelle, R. Songmuang, B. Daudin, B. Gayral, and M. Kociak, "Lifetime Measurements Well below the Optical Diffraction Limit," ACS Photonics 3, 1157-1163 (2016)

${ }^{23}$ S. Meuret, T. Coenen, S. Y. Woo, Y. H. Ra, Z. Mi, and A. Polman, "Nanoscale Relative Emission Efficiency Mapping Using Cathodoluminescence g(2) Imaging," Nano Letters 18, 22882293 (2018).

${ }^{24}$ S. Meuret, M. Solà Garcia, T. Coenen, E. Kieft, H. Zeijlemaker, M. Lätzel, S. Christiansen, S. Y. Woo, Y. H. Ra, Z. Mi, and A. Polman, "Complementary cathodoluminescence lifetime imaging configurations in a scanning electron microscope," Ultramicroscopy 197, 28-38 (2019).

${ }^{25}$ R. Koester, J. S. Hwang, C. Durand, D. Le Si Dang, and J. Eymery, "Self-assembled growth of catalyst-free GaN wires by metal-organic vapour phase epitaxy," Nanotechnology 21 , $015602(2010)$

${ }^{26}$ A. Kapoor, S. Finot, V. Grenier, E. Robin, C. Bougerol, J. Bleuse, G. Jacopin, J. Eymery, and C. Durand, "Role of Underlayer for Efficient Core-Shell InGaN QWs Grown on m -plane GaN Wire Sidewalls," ACS Applied Materials \& Interfaces 12 19092-19101 (2020)

${ }^{27}$ D. V. Dinh, N. Hu, Y. Honda, H. Amano, and M. Pristovsek, "Aluminium incorporation in polar, semi- and non-polar AlGaN layers: a comparative study of x-ray diffraction and optical properties," Scientific Reports 9, 15802 (2019).

${ }^{28}$ K. Hestroffer, R. Mata, D. Camacho, C. Leclere, G. Tourbot, Y. M. Niquet, A. Cros, C. Bougerol, H. Renevier, and B. Daudin, "The structural properties of GaN/AlN core-shell nanocolumn heterostructures," Nanotechnology 21, 415702 (2010).

${ }^{29}$ S. Figge, H. Kröncke, D. Hommel, and B. M. Epelbaum, "Temperature dependence of the thermal expansion of AlN," Applied Physics Letters 94, 22-25 (2009).

${ }^{30}$ D. Drouin, A. R. Couture, D. Joly, X. Tastet, V. Aimez, and R. Gauvin, "CASINO V2.42-A Fast and Easy-to-use Modeling Tool for Scanning Electron Microscopy and Microanalysis Users," Scanning 29, 92-101 (2007).

${ }^{31}$ S. Birner, T. Zibold, T. Andlauer, T. Kubis, M. Sabathil, A. Trellakis, and P. Vogl, "nextnano: General Purpose 3-D Simulations," IEEE Transactions on Electron Devices 54, 2137-2142 (2007)

${ }^{32}$ I. Vurgaftman and J. R. Meyer, "Band parameters for nitrogencontaining semiconductors," Journal of Applied Physics 94, 3675-3696 (2003)

${ }^{33}$ C. Frankerl, F. Nippert, M. P. Hoffmann, H. Wang, C. Brandl, H.-J. Lugauer, R. Zeisel, A. Hoffmann, and M. J. Davies, "Strongly localized carriers in Al-rich AlGaN/AlN single quantum wells grown on sapphire substrates," Journal of Applied Physics 127, 095701 (2020).

${ }^{34}$ V. M. Kaganer, J. Lähnemann, C. Pfüller, K. K. Sabelfeld, A. E. Kireeva, and O. Brandt, "Determination of the Carrier Diffusion Length in Ga N from Cathodoluminescence Maps Around Threading Dislocations: Fallacies and Opportunities," Physical Review Applied 12, 1 (2019)

${ }^{35}$ T. Kawashima, H. Yoshikawa, S. Adachi, S. Fuke, and K. Ohtsuka, "Optical properties of hexagonal GaN," Journal of Applied Physics 82, 3528-3535 (1997).

${ }^{36} \mathrm{~L}$. Rigutti, G. Jacopin, L. Largeau, E. Galopin, A. De Luna Bugallo, F. H. Julien, J.-C. Harmand, F. Glas, and M. Tchernycheva, "Correlation of optical and structural properties of GaN/AlN core-shell nanowires," Physical Review B 83, 155320 (2011).

${ }^{37}$ G. Jacopin, L. Rigutti, S. Bellei, P. Lavenus, F. H. Julien, A. V. Davydov, D. Tsvetkov, K. A. Bertness, N. A. Sanford, J. B. 
Schlager, and M. Tchernycheva, "Photoluminescence polarization in strained GaN/AlGaN core/shell nanowires," Nanotechnology 23, 325701 (2012).
${ }^{38}$ M. Fouchier, N. Rochat, E. Pargon, and J. P. Landesman, "Polarized cathodoluminescence for strain measurement," Review of Scientific Instruments 90, 043701 (2019). 


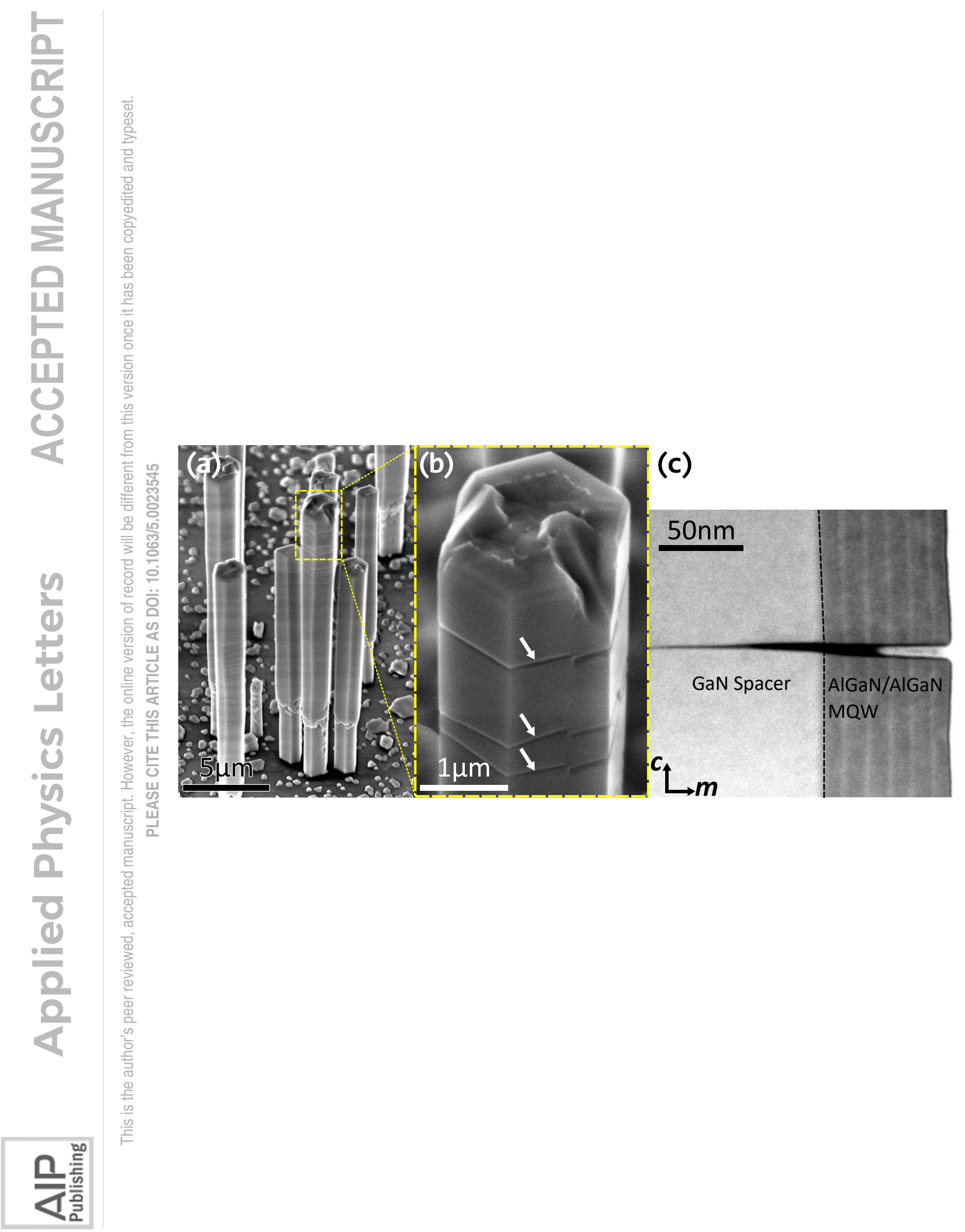




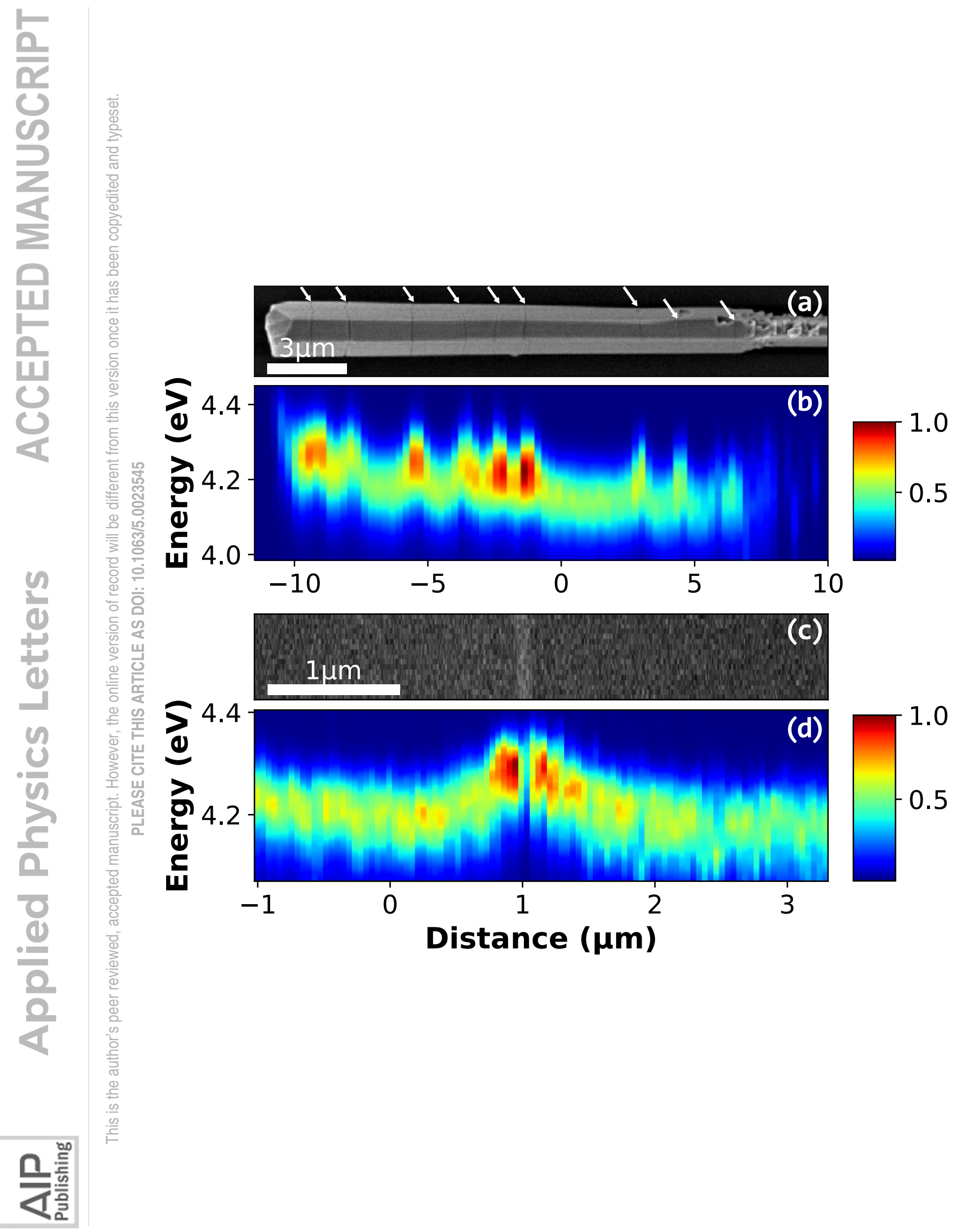




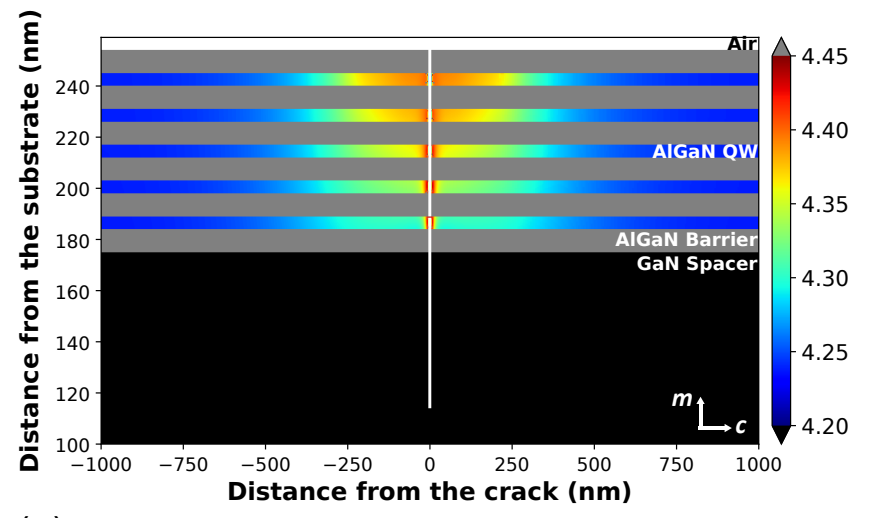

(a)

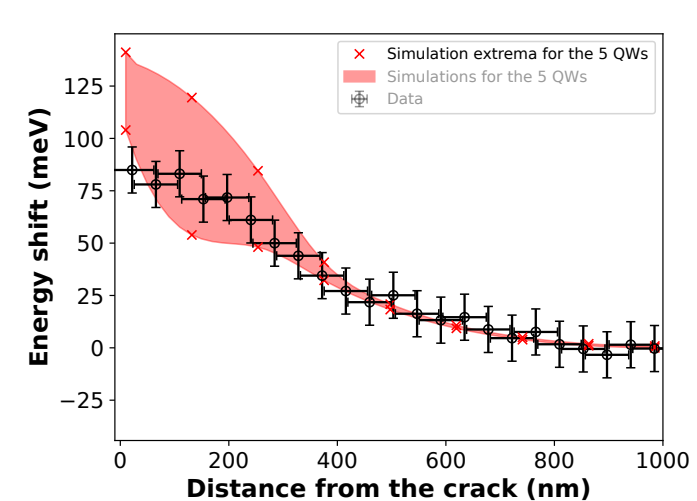

(b) 


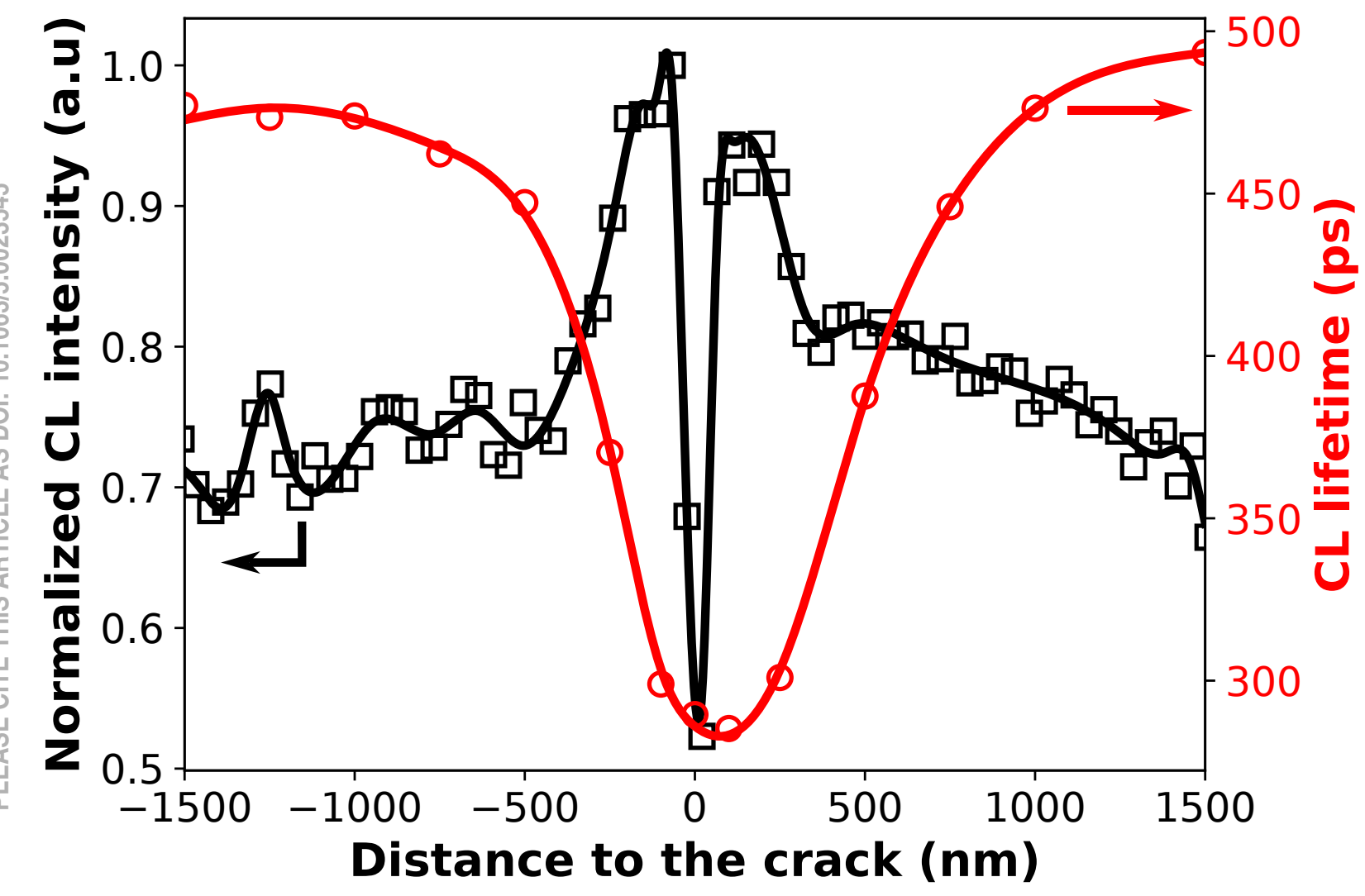

